\title{
Assessment of Undiscovered Conventional Oil and Gas Resources of South America and the Caribbean, 2012
}

Using a geology-based assessment methodology, the U.S. Geological Survey estimated means of 126 billion barrels of oil and 679 trillion cubic feet of undiscovered natural gas in 31 geologic provinces of South America and the Caribbean.

\section{Introduction}

The U.S. Geological Survey (USGS) assessed the potential for undiscovered conventional oil and gas fields within priority geologic provinces of South America and the Caribbean as part of the USGS World Petroleum

Resources Project (fig. 1). Thirty-one geologic provinces were assessed in this study, which represents a complete re-assessment of the South America-Caribbean region published in 2000 (U.S. Geological Survey World Energy Assessment Team, 2000).

The methodology for the assessment included a complete geologic framework description for each province based mainly on published literature and definition of petroleum systems and assessment units (AU) within these systems. Exploration and discovery history was a critical part of the methodology to determine sizes and numbers of undiscovered accumulations. In those AUs with few or no discoveries, geologic and production analogs were used to estimate sizes and numbers of undiscovered oil and gas accumulations. Each assessment unit was assessed for undiscovered oil and nonassociated gas accumulations, and co-product ratios were used to calculate the volumes of associated gas (gas in oil fields) and volumes of natural gas liquids. This assessment is for conventional oil and gas resources only; unconventional resource assessments (shale gas, shale oil, tight gas) for this region are being completed in a separate study.

The provinces assessed in this study represent a wide range of tectonic settings and evolution, stratigraphic fill, thermal evolution, petroleum systems, and exploration history. There are many provinces in this region that have been maturely explored, such as Maracaibo Basin, Neuquen Basin, Magallanes

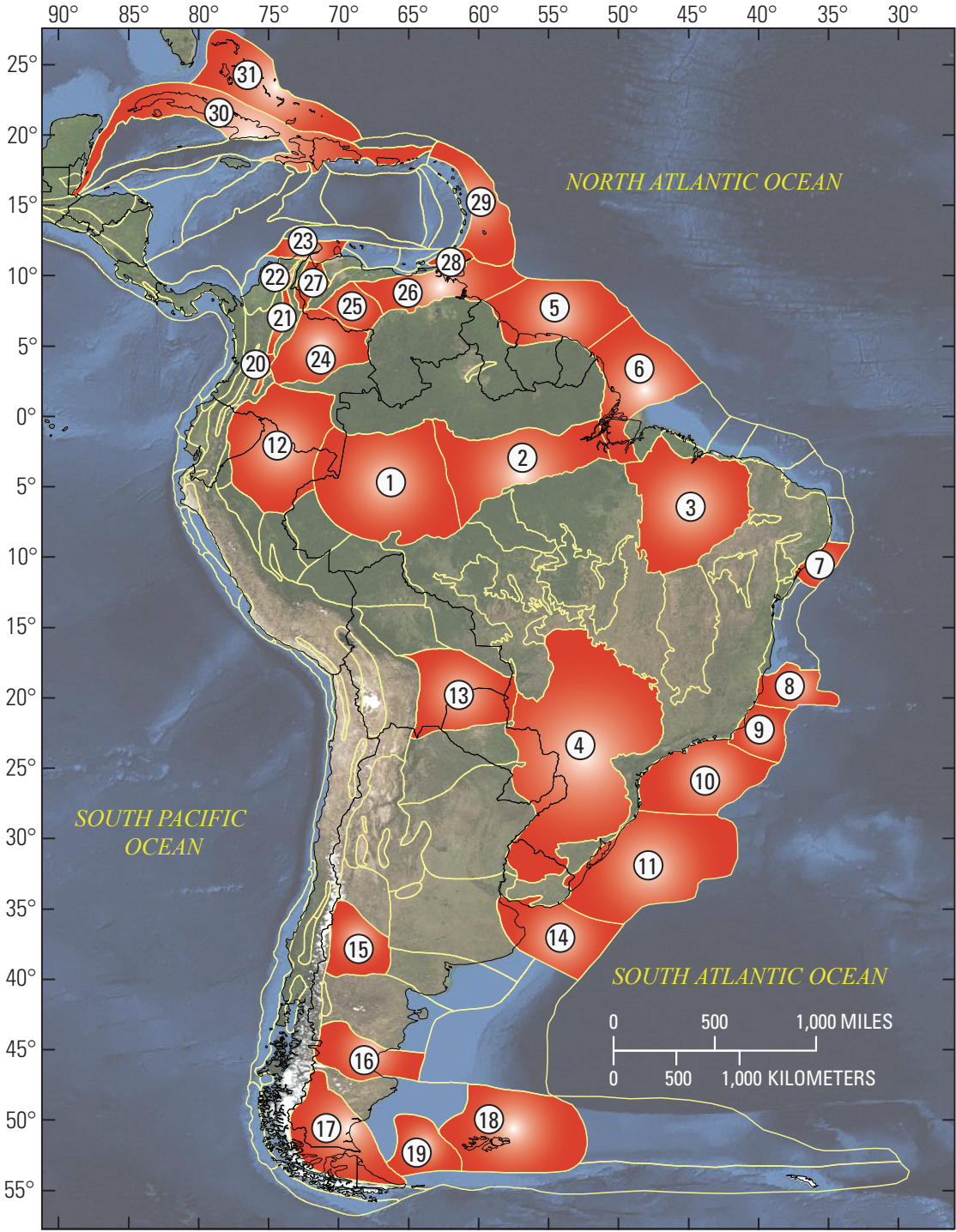

Figure 1. Locations of 31 provinces of South America and Caribbean assessed in this study. Province numbers and names are included in table 1.

Basin, and Llanos Basin, whereas several provinces have few or no discoveries, such as Salado-Punta del Este, Parnaiba, and Bahama Platform. The range of resource estimates for each province reflects the geologic uncertainty.

\section{Resource Summary}

The USGS assessed undiscovered conventional oil and gas resources in assessment units within 31 geologic provinces (table 1). For undiscovered resources, the mean totals are as follows: (1) 125,900 million barrels of oil (MMBO), with a range from 44,556 to 261,862 MMBO; (2) 678,537 billion cubic feet of gas (BCFG), with a range from 229,547 to 1,476,008 $\mathrm{BCFG}$; and (3) 21,001 million barrels of natural gas liquids (MMBNGL), with a range from 6,850 to 46,581 MMBNGL. 
Table 1. South America and Caribbean assessment results for undiscovered, technically recoverable oil, gas, and natural gas liquids.

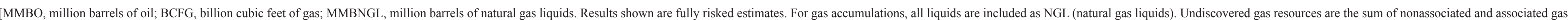
F95 represents a 95-percent chance of at least the amount tabulated; other fractiles are defined similarly. Fractiles are additive under assumption of perfect positive correlation. Gray shading indicates not applicable]

\begin{tabular}{|c|c|c|c|c|c|c|c|c|c|c|c|c|c|c|c|}
\hline \multirow{2}{*}{$\begin{array}{c}\text { Map } \\
\text { location } \\
\text { number }\end{array}$} & \multirow{2}{*}{ Province name } & \multirow{2}{*}{$\begin{array}{l}\text { Province } \\
\text { code }\end{array}$} & \multirow{2}{*}{$\begin{array}{l}\text { Field } \\
\text { type }\end{array}$} & \multicolumn{8}{|c|}{$\begin{array}{l}\text { Total undiscovered resources } \\
\text { Gas (BCFG) }\end{array}$} & \multicolumn{4}{|c|}{ NGL (MMBNGL) } \\
\hline & & & & F95 & F50 & F5 & Mean & F95 & F50 & F5 & Mean & F95 & F50 & F5 & Mean \\
\hline (1) & & & & 260 & 536 & 1,018 & 574 & 2,108 & 4,358 & 8,316 & 4,673 & 45 & 104 & 229 & 116 \\
\hline (1) & Solimoes Basin & 6011 & Gas & & & & & 9,420 & 17,317 & 31,363 & 18,454 & 260 & 478 & 867 & 509 \\
\hline (2) & Amazonas Basin & 6012 & Oil & 130 & 330 & 742 & 369 & 1,057 & 2,684 & 6,061 & 3,005 & 22 & 63 & 168 & 75 \\
\hline & Amazonas Basin & 6012 & Gas & & & & & 2,709 & 6,024 & 12,734 & 6,648 & 75 & 166 & 351 & 184 \\
\hline (3) & Parnaiba Basin & 6016 & Oil & 1,397 & 2,786 & 5,201 & 2,972 & 11,357 & 22,662 & 42,414 & 24,197 & 247 & 542 & 1,137 & 598 \\
\hline & Parnalba Basin & 6010 & Gas & & & & & 8,342 & 16,628 & 31,114 & 17,759 & 230 & 459 & 860 & 490 \\
\hline (4) & Parana Basin & 6020 & Oil & 188 & 400 & 770 & 429 & 1,527 & 3,250 & 6,275 & 3,492 & 32 & 76 & 175 & 86 \\
\hline & & & $\begin{array}{l}\text { Gas } \\
\text { Oil }\end{array}$ & 5167 & 12494 & 25981 & 13608 & 3,240 & $\begin{array}{r}6,215 \\
18284\end{array}$ & 11,364 & $\begin{array}{r}6,610 \\
21,10 .\end{array}$ & 140 & 272 & 502 & 289 \\
\hline (5) & Guyana-Suriname Basin & 6021 & Gas & & 12,494 & & & 0,253 & $\begin{array}{l}10,04 \\
9,887\end{array}$ & $\begin{array}{ll}40,200 \\
20,664\end{array}$ & $\begin{array}{l}2,1,100 \\
10,836\end{array}$ & 100 & 262 & $\begin{array}{l}1,244 \\
561\end{array}$ & $\begin{array}{l}584 \\
289\end{array}$ \\
\hline 6 & Foz do Amazonas Basin & 6022 & Oil & 201 & 583 & 1,511 & 684 & 251 & 848 & 2,638 & 1,075 & 7 & 23 & 73 & 29 \\
\hline & Fuz du Alitazulias Dasmir & & Gas & & 1684 & & & 16,136 & 31,742 & 58,701 & 33,786 & 813 & 1,600 & 2,963 & 1,704 \\
\hline (7) & Sergipe-Alagoas Basin & 6029 & Gas & 561 & 1,684 & 4,425 & 1,986 & $\frac{1,203}{644}$ & $\frac{3,683}{2,704}$ & $\frac{10,227}{11729}$ & $\begin{array}{l}4,435 \\
3.902\end{array}$ & $\frac{72}{38}$ & $\frac{223}{163}$ & $\begin{array}{l}635 \\
709 \\
\end{array}$ & $\frac{271}{236}$ \\
\hline 8 & Espirito Santo Basin & 6034 & Oil & 334 & 1,329 & 5,508 & 1,908 & 638 & 2,697 & 11,840 & 4,011 & 10 & 49 & 235 & 76 \\
\hline & 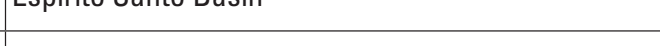 & ( & Gas & & & & & 3,285 & 10,983 & 39,831 & 14,803 & 107 & 386 & 1,485 & 536 \\
\hline (9) & Campos Basin & 6035 & $\begin{array}{lll}\text { Oil } \\
\text { Gas }\end{array}$ & 1,924 & 7,807 & 44,836 & 14,736 & 1,192 & 10,256 & 95,243 & 27,560 & 28 & 297 & 2,896 & 829 \\
\hline 2 & & & Oil & 23839 & 54,434 & 113,392 & 59,689 & $\begin{array}{r}115 \\
56,405\end{array}$ & $\begin{array}{r}1,142 \\
131477\end{array}$ & $\begin{array}{r}22,502 \\
291722\end{array}$ & $\begin{array}{r}5,302 \\
147515\end{array}$ & $\begin{array}{r}4 \\
1.668\end{array}$ & $\begin{array}{r}36 \\
3853\end{array}$ & $\frac{703}{8.514}$ & $\begin{array}{r}167 \\
4,309\end{array}$ \\
\hline (10) & Santos Basin & 6036 & Gas & & & & & 10,203 & $\begin{array}{l}\frac{10,441}{43,103} \\
\end{array}$ & $\frac{292,1 / 26}{1898}$ & $\frac{14,1305}{62,305}$ & 3002 & $\begin{array}{l}1,050 \\
1,284 \\
\end{array}$ & $\begin{array}{l}0,7,44 \\
5,448 \\
\end{array}$ & $\begin{array}{l}4,009 \\
1,857\end{array}$ \\
\hline (11) & Pelotas Basin & 6037 & Oil & 244 & 664 & 1,736 & 782 & 557 & 1,526 & 4,090 & 1,817 & 34 & 93 & 249 & 110 \\
\hline & & & Gas & & & & & 8,104 & 17,505 & 34,141 & 18,817 & 173 & 378 & 754 & 410 \\
\hline (12) & Putamayo-Oriente-Maranon Basin & 6041 & Oil & 3,157 & 4,585 & 6,633 & 4,698 & 1,411 & 2,092 & 3,061 & 2,144 & 37 & 56 & 82 & 57 \\
\hline (1) & 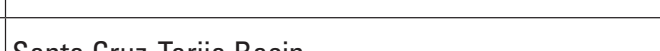 & & Oil & 60 & 111 & 196 & 118 & $\frac{0}{249}$ & $\begin{array}{r}0 \\
475\end{array}$ & $\begin{array}{r}0 \\
866\end{array}$ & $\begin{array}{r}0 \\
505\end{array}$ & 0 & 0 & $\frac{0}{19}$ & 0 \\
\hline (13) & Santa Cruz-Tarija Basin & 6045 & Gas & & & & & 11,091 & 24,012 & $\begin{array}{r}8060 \\
47,595\end{array}$ & 26,016 & 468 & 1,033 & $\begin{aligned} 19 \\
2,156\end{aligned}$ & $\frac{11}{1,137}$ \\
\hline (14) & Salado-Punta del Este Basin & 6054 & Oil & 643 & 1,752 & 4,064 & 1,975 & 1,471 & 4,058 & 9,503 & 4,593 & 89 & 246 & 580 & 279 \\
\hline & & & Gas & 153 & 311 & 591 & 321 & 8,129 & 19,910 & 43,735 & 22,180 & 173 & 432 & 960 & 482 \\
\hline (15) & Neuquen Basin & 6055 & Gas & 153 & 311 & 591 & 334 & $\frac{206}{1,511}$ & $\begin{array}{r}446 \\
3,427\end{array}$ & $\begin{array}{r}955 \\
7,230\end{array}$ & $\begin{array}{r}497 \\
3777\end{array}$ & $\frac{4}{23}$ & $\frac{9}{53}$ & $\frac{21}{116}$ & $\frac{10}{59}$ \\
\hline (16) & San Jorae Basin & 6058 & Oil & 33 & 63 & 116 & 67 & 23 & 46 & 93 & 51 & 0 & 1 & 2 & $\frac{3}{1}$ \\
\hline & Jan Jorge basin & 0058 & Gas & & & & & 95 & 195 & 373 & 210 & 2 & 4 & 7 & 4 \\
\hline (17) & Magallanes Basin & 6059 & Oil & 60 & 163 & 501 & 205 & 134 & 379 & 1,249 & 497 & 2 & 5 & 15 & 6 \\
\hline & & & Gas & 1,157 & & & & 2,747 & 5,510 & 10,412 & 5,896 & 73 & 159 & 339 & 176 \\
\hline (18) & Falklands Plateau & 6060 & OII & 1,457 & 4,413 & 12,224 & 5,302 & 3,215 & $\begin{array}{l}9,837 \\
2025\end{array}$ & 28,575 & 12,060 & 40 & 125 & 372 & 154 \\
\hline & & & Gas & 38 & 118 & 307 & 138 & $\frac{10,584}{85}$ & $\frac{32,885}{277}$ & 88,3166 & $\frac{38,940}{333}$ & $\frac{25 /}{1}$ & $\frac{820}{3}$ & $\frac{2,478}{10}$ & 1,024 \\
\hline (19) & Malvinas Basin & 6063 & Gas & & & & & 2,560 & $\frac{2 \pi l}{5,394}$ & $\frac{114}{10,227}$ & $\begin{array}{l}3,75 \\
5,600\end{array}$ & $\frac{1}{67}$ & $\frac{5}{156}$ & $\frac{10}{333}$ & $\begin{array}{r}4 \\
172\end{array}$ \\
\hline 20 & Upper Magdelena Basin & 6089 & Oil & 163 & 357 & 729 & 390 & 225 & 502 & 1,077 & 556 & 5 & 10 & 22 & 11 \\
\hline & & & Gas & & & & & 81 & 358 & 1,424 & 499 & 1 & 5 & 22 & 8 \\
\hline (21) & Middle Magdelena Basin & 6090 & Oil & 238 & 495 & 959 & 534 & 384 & 840 & 1,850 & 942 & 6 & 13 & 29 & 15 \\
\hline ? & & & Gas & 15 & 66 & 286 & 97 & $\frac{131}{45}$ & 490 & $\begin{array}{r}1,831 \\
867\end{array}$ & 666 & 2 & 7 & 28 & 10 \\
\hline (स) & Lower Mlagdelena Basin & 6091 & Gas & 13 & 00 & 200 & II & 971 & 2,584 & 6,211 & 2,951 & $\frac{1}{70}$ & $\begin{array}{r}4 \\
189 \\
\end{array}$ & $\begin{array}{l}10 \\
470\end{array}$ & 218 \\
\hline (23) & Guarija Basin & 6095 & Oil & 6 & 36 & 208 & 63 & 18 & 108 & 629 & 190 & 0 & 2 & 13 & 4 \\
\hline & & & Gas & 957 & 2418 & 5215 & $2005=$ & $\begin{array}{r}578 \\
1702\end{array}$ & 1,982 & 5,554 & 2,385 & 12 & 41 & 118 & 50 \\
\hline (24) & Llanos Basin & 6096 & Gas & 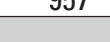 & 2,418 & 5,215 & 2,665 & 1,702 & $\begin{array}{l}4,545 \\
2,250 \\
250\end{array}$ & $\frac{11,008}{0}$ & 5,219 & 55 & 151 & 376 & 175 \\
\hline (25) & Barinas-Anure Basin & 6007 & Oil & 90 & 398 & 1,567 & 551 & 7 & 2,350 & 8,899 & 3,184 & 61 & 245 & $\frac{943}{2}-x-10$ & 338 \\
\hline & Barinas-Apure Basin & 年 & Gas & & & & & 42 & 400 & 3,447 & 887 & 0 & 4 & $\frac{2}{35}$ & 9 \\
\hline (26) & East Venezuela Basin & 6098 & Oil & 1,138 & 2,944 & 7,126 & 3,379 & 3,837 & 9,914 & 24,081 & 11,410 & 82 & 214 & 538 & 249 \\
\hline & & & Gas & 340 & 838 & 1822 & 929 & $\begin{aligned} 11,269 \\
637\end{aligned}$ & 26,387 & 55,483 & $\frac{28,938}{1959}$ & 301 & 729 & 1,605 & 810 \\
\hline (27) & Maracaibo Basin & 6099 & Gas & & & & & $\frac{0.1}{1,584}$ & $\frac{1,0000}{4,938}$ & $\begin{array}{l}4,1,90 \\
13,934\end{array}$ & $\begin{array}{l}5,969 \\
5,969\end{array}$ & $\frac{20}{45}$ & 142 & 401 & $\frac{80}{172}-3 x-3$ \\
\hline 28 & Tobago Trough & 6103 & Oil & 0 & 0 & 0 & 0 & 0 & 0 & 0 & 0 & 0 & 0 & 0 & 0 \\
\hline & & 0100 & Gas & & & & & 6,558 & 14,554 & 29,048 & 15,742 & 22 & 54 & 140 & 65 \\
\hline (29) & Barbados Accretionary Prism & 6107 & Oil & 22 & 92 & 498 & 154 & 33 & 168 & 1,028 & 310 & 1 & 6 & 39 & 12 \\
\hline 30 & North Cuba Basin of Greater Antilles Deformed Belt & & Oil & 1,185 & 4,301 & 9,364 & 4,660 & 1,865 & $\frac{7,4000}{7,558}$ & 17,961 & $\frac{74,399}{8,432}$ & 163 & 709 & 1,825 & 814 \\
\hline & North Cuba Basin of Greater Antilles Deformed Belt & 6117 & Gas & & & & & 141 & 862 & 3,418 & 1,190 & 7 & 44 & 185 & 63 \\
\hline 31 & Bahamas Platform & & oil & 554 & 1,591 & 4,336 & 1,906 & 692 & 2,028 & 5,736 & 2,467 & 42 & 126 & 362 & 155 \\
\hline (II) & Bahamas Plattorm & 6719 & Gas & & & & & 705 & 2,723 & 12,170 & 4,051 & 11 & 45 & 212 & 70 \\
\hline & Total conventional resources & & & 44,556 & 108,098 & 261,862 & 125,900 & 229,547 & 571,684 & $1,476,008$ & 678,537 & 6,850 & 17,507 & 46,581 & 21,001 \\
\hline
\end{tabular}


Of the mean oil total of $125,900 \mathrm{MMBO}$, about 44 percent $(55,601 \mathrm{MMBO})$ is estimated to be in subsalt reservoirs in the Santos, Campos, and Espirito Santo Basin Provinces, with most undiscovered subsalt oil estimated to be in the Santos Basin Province. In addition, several provinces are estimated to have significant undiscovered conventional oil potential, including the Guyana-Suriname Basin Province (mean of 13,608 MMBO); Falklands Plateau Province (mean of 5,302 MMBO); Putumayo-Oriente-Maranon Basin Province (mean of 4,698 MMBO); North Cuba Basin Province (mean of 4,660 MMBO); and the Parnaiba Basin Province (mean of 2,972 MMBO).

For the mean undiscovered conventional gas total of $678,537 \mathrm{BCFG}$, about 55 percent $(374,956 \mathrm{BCFG})$ is estimated to be in five provinces: (1) Santos Basin Province (mean of 209,820 BCFG); (2) Falklands Plateau Province (mean of 51,000 BCFG), (3) Parnaiba Basin Province (mean of 41,956 BCFG), (4) East Venezuela Basin Province (mean of 40,348 BCFG), and (5) Guyana-Suriname Basin Province (mean of 32,032 BCFG).

\section{Reference Cited}

\section{U.S. Geological Survey World}

Energy Assessment Team, 2000, U.S. Geological Survey world petroleum assessment 2000-Description and results: U.S. Geological Survey Digital Data Series DDS-60, 4 CD-ROMs.

\section{For Further Information}

Supporting studies of the geologic models and the methodology used in the assessment of South America and Caribbean provinces are in progress. Assessment results are available at the USGS Energy Program website, http://energy.usgs.gov/oilgas/.

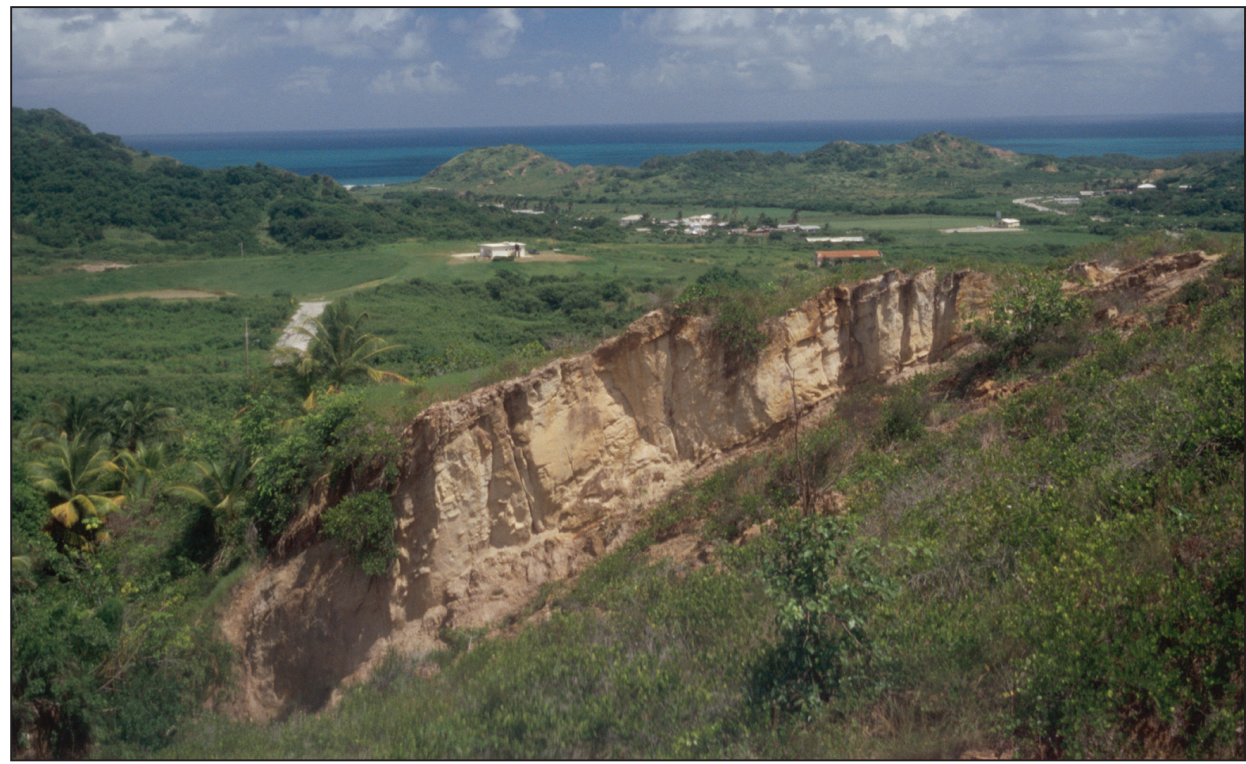

Eocene turbidites of the Scotland District, Barbados. Photograph by C.J. Schenk, U.S. Geological Survey, 2011.

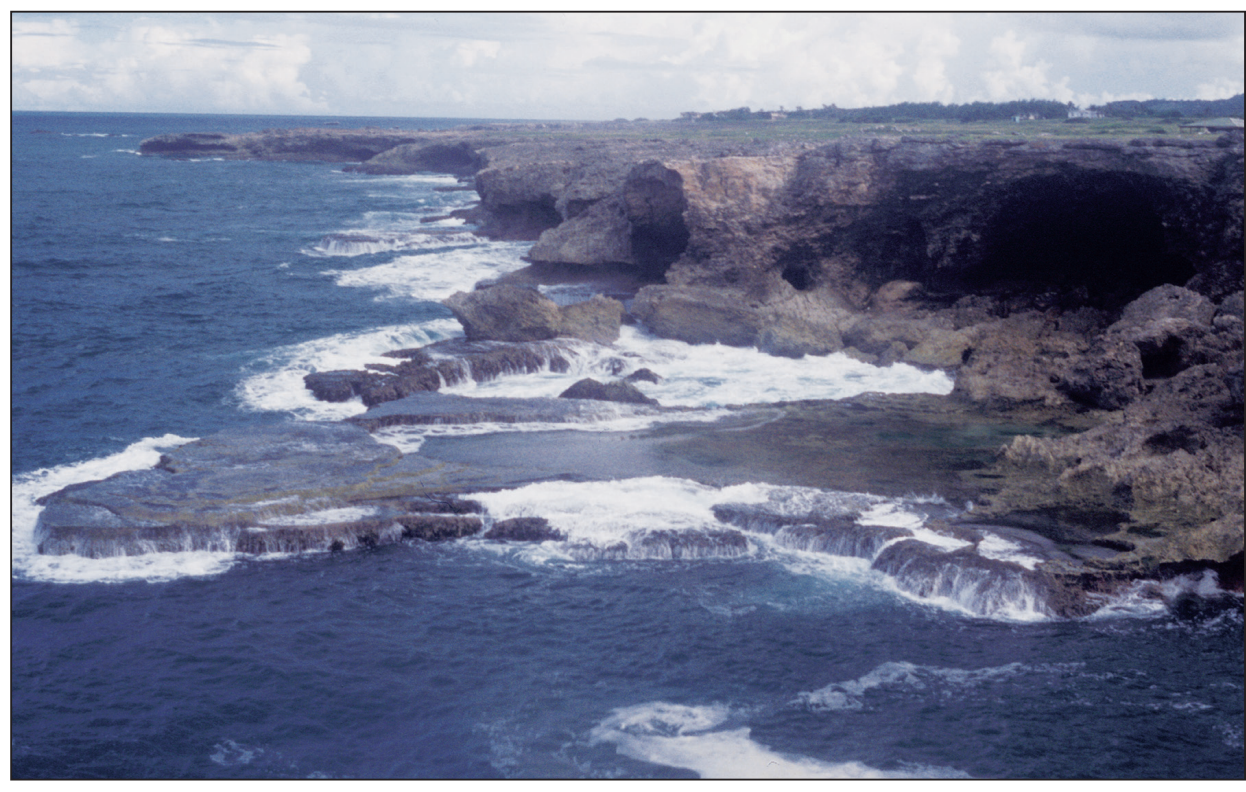

Cliffs of carbonate rock along the east coast of the island of Barbados. Photograph by C.J. Schenk, U.S. Geological Survey, 2011.

\section{South America-Caribbean Assessment Team}

Christopher J. Schenk, Michael E. Brownfield, Ronald R. Charpentier, Troy A. Cook, Timothy R. Klett, Mark A. Kirschbaum, Janet K. Pitman, Richard M. Pollastro, and Marilyn E. Tennyson. 